\title{
DO SERVIÇO SOCIAL MÉdico AO SERVIÇO SOCIAL NA SAÚdE: MEIO SÉCULO DE HISTÓRIA EM SÃO PAULO
}

FROM MEDICAL SOCIAL WORK TO SOCIAL WORK IN HEALTH CARE:

HALF ACENTURY OF HISTORY IN SÃO PAULO

\author{
Regina Maria Giffoni Marsiglia ${ }^{1}$ \\ Maria Olinda Costa Santos Carreira ${ }^{2}$ \\ Denise Perroud Amaral ${ }^{3}$
}

\section{RESUMO}

Este artigo se propõe a apresentar a inserção do Serviço Social e dos Assistentes Sociais na área de saúde em São Paulo, nas últimas seis décadas. Parte da perspectiva de que estudos de caráter regional são fundamentais para a produção do conhecimento sobre processos sociais complexos em Formações Históricas e Sociais, dadas à diversidade e heterogeneidade das relações econômicas, políticas e sociais que se instalaram historicamente, nas diversas regiões do país. Partiu-se da identificação de relações entre a Assistência Social e a Saúde desde o século XIX, da definição de suas áreas específicas durante a primeira metade do século XX, e da profissionalização do Serviço Social a partir da fundação da Escola de Serviço Social em São Paulo, em 1936. Utilizou-se de pesquisa bibliográfica, bem como de pesquisa empírica sobre os Trabalhos de Conclusão de Curso (TCC) sobre a temática da saúde, apresentados entre 19401999, pelos formandos na graduação de Serviço Social da Pontifícia Universidade Católica de São Paulo (PUCSP) e das Faculdades Metropolitanas Unidas (FMU), ambas localizadas na capital do Estado de São Paulo. Essa produção, ao registrar e analisar a prática de estágio dos alunos em serviços de saúde permite acessar as formas de inserção, lócus institucional, temas e preocupações dos assistentes sociais nos hospitais e na Atenção Primária em Saúde. A produção mais recente da categoria profissional, apresentada através de relatórios de eventos, revistas e livros, complementa o conhecimento sobre as articulações entre o Serviço Social e a Saúde em São Paulo.

PALAVRAS-CHAVE: Serviço Social. Saúde. São Paulo

\footnotetext{
${ }^{1}$ Assistente Doutora do Programa de Estudos Pós-Graduados em Serviço Social da Pontifícia Universidade Católica de São Paulo e Professora Adjunta da Faculdade de Ciências Médicas da Santa Casa de São Paulo- FCMSCSP- Regina.marsiglia@fcmsantacasasp.edu.br. Fone: 551133677760

${ }^{2}$ Doutora em Serviço Social pela PUCSP, com experiência em Atenção Primária na Saúde na SMSSP. 3 Mestre em Serviço Social pela PUCSP e Assistente Social do Centro de Saúde Escola "Alexandre Vranjac” da ISCMSP e FCMSCSP.
} 


\begin{abstract}
The proposal of this article is to present how Social Work and Social Assistants have been inserted in Health Care in São Paulo in the last six decades. It is based on the perspective that studies of regional nature are fundamental for the production of knowledge on complex social processes in Historical and Social Formations, given the diversity and heterogeneity of the economic, political, and social relations that have historically been installed in the different regions of the country. We started by identifying the relationship between Social Assistance and Health Care since the $19^{\text {th }}$ century, by defining its specific areas in the first half of the $20^{\text {th }}$ century, and by considering the professionalization of Social Work with the foundation of the Escola de Serviço Social (School of Social Work) in São Paulo, in 1936. We used bibliographic research, as well as empirical research on the TCCs - Trabalhos de Conclusão de Curso (Course Completion Works) on the theme of health care presented between 1940 and 1999 by the graduating Social Work students of Pontifícia Universidade Católica de São Paulo (PUCSP) and of Faculdades Metropolitanas Unidas (FMU), both located in the capital city of the State of São Paulo. By registering and analyzing the students' internship practices in health care services, this production allowed us to have access to the forms of insertion, institutional locus, themes and concerns of the social assistants in the hospitals and the Primary Health Care. The knowledge about the articulations between Social Work and Health Care in São Paulo was complemented by the latest production of the professional category, presented through event reports, magazines and books.
\end{abstract}

KEYWORDS: Social Work. Health Care. São Paulo.

\title{
INTRODUÇÃO
}

A saúde emerge com a questão social para a economia cafeeira de exportação no final do século XIX, na tentativa de controle das endemias na área rural e do saneamento dos centros urbanos e dos portos do Rio de Janeiro, Santos e Recife. Com a chegada da peste bubônica a partir de 1899, passamos a seguir o programa internacional de saneamento marítimo, por imposição dos países capitalistas centrais e, visando garantir o complexo exportador e o capital industrial nascente (BRAGA; PAULA, 1981; IYDa,1994).

As medidas tomadas pelo poder público para enfrentar doenças como a cólera, peste bubônica, febre amarela, varíola e as doenças infecciosas e parasitárias foram as edificações de obras de saneamento do meio ambiente, campanhas sanitárias, medidas jurídicas impostas de vacinação e isolamento compulsório dos doentes. Apesar dessas medidas, os maiores recursos no início do século foram destinados à assistência médico hospitalar, desenvolvida a nível privado, no interior das famílias ou pelas entidades 
filantrópicas mantidas por contribuições de proprietários rurais e subvenções estatais (IYDA, 1994).

As pressões internacionais para adoção de medidas de massa foram muito fortes: os países europeus em 1851, na I Conferência Internacional de Saúde em Paris, decidiram adotar medidas comuns contra a disseminação da peste, cólera e febre amarela, pressionando nas décadas posteriores, os demais países para fazerem o mesmo, principalmente os países que dependiam da imigração estrangeira, como o Brasil.

Assumindo a direção do Serviço Sanitário Estadual em São Paulo no início do século XX, já durante a década de 20, Paula Souza passou a defender a cloração da água para controlar as diarreias e a febre tifoide que se expandia na capital paulista, a criação dos centros de saúde, enquanto serviços ambulatoriais permanentes, e a ênfase nos programas de educação sanitária. Posteriormente, seu sucessor na direção do órgão sanitário em 1927, propôs que o poder público procurasse desenvolver uma ação coordenada de saúde pública e de assistência social, pois no seu entender, as medidas sanitárias mais eficientes eram aquelas que combatiam a pobreza e os pobres (MERHY, 1985).

Percebe-se aqui uma clara alusão à importância da Assistência Social para a Saúde, ações de assistência social, que eram exercidas em geral por médicos e leigos, principalmente mulheres, que se dedicavam à filantropia, em associações ou ligas (implantadas desde o período imperial) contra a lepra e tuberculose, e de proteção à infância; e principalmente por organizações da sociedade civil, em especial da Igreja Católica, e pelas Irmandades de Misericórdia, instituições portuguesas transplantadas para o Brasil e que cuidavam dos grupos marginalizados na sociedade; e posteriormente, pelas associações beneficentes que passaram a ganhar maior importância no país, no atendimento das necessidades dos imigrantes (MERHY,1985; IYDA, 1994).

A presença do Estado dava-se por meio de subvenções aos hospitais filantrópicos, pela assistência direta aos seus funcionários civis e militares e manutenção dos hospitais de isolamento, dos hospitais para doentes mentais e dos leprosários públicos, através do Serviço Sanitário do Estado (PALMA, 1996).

A alta mortalidade infantil na Capital de São Paulo foi também preocupação desse período, justificando o estudo de suas causas já em 1882, por uma comissão Serv. Soc. \& Saúde, Campinas, SP v.15, n. 1 (21), p. 37-50, jan./jun. 2016 ISSN 1676-6806 
criada pelo Secretário de Negócios do Interior. O relatório final apontou como principais causas, os nascimentos ilegítimos, alimentação precária, habitações insalubres, abuso do álcool, falta de asseio e as oscilações de temperatura e umidade da Capital. Foram apontadas ainda as dificuldades de adaptação dos imigrantes e suas famílias à alimentação local, bem como os poucos recursos que os operários dispunham para sobreviver, exigindo muitas vezes que as mães trabalhassem fora interrompendo o aleitamento natural, e obrigando as crianças a receber alimentos destinados aos adultos desde a mais tenra idade.

A Liga Paulista contra a Tuberculose criou em 1904, um Dispensário, para atendimento dos "deserdados da sorte, os vencidos da vida", cujas funções além da distribuição de medicamentos, abrangiam a distribuição de alimentos, o auxílio para pagamento de aluguel, visitas domiciliares para orientação às famílias dos doentes pobres. Seus dirigentes associavam a tuberculose à miséria e consideravam que a recuperação do doente dependia de suas condições de vida. A campanha contra tuberculose centrou sua ação no doente e sua família, associando ações de assistência médica e assistência social, apelando para o sentimento de caridade cristã da elite paulista, principalmente das esposas e filhas dos grandes proprietários, industriais e políticos. Por isso diferiu muito da Campanha contra a Febre Amarela, assumida exclusivamente pelo Serviço Sanitário, e centrada no combate ao mosquito transmissor, através de ações sobre meio ambiente.

A inauguração do novo hospital da Santa Casa de Misericórdia de São Paulo junto com uma Casa dos Expostos para receber recém-nascidos abandonados, em 1825 no Largo dos Ingleses, foi na assistência hospitalar de São Paulo um marco importante. Mas suas dependências logo se tornaram insuficientes, obrigando à construção de outro prédio na Rua da Glória em 1840, também como hospital e Casa dos Expostos, confirmando a prática de articulação da assistência médica com a assistência social nesse período. Nessa década já existiam mais três hospitais no interior da então província de São Paulo. Outro hospital inaugurado em 1876 foi o Hospital da Beneficência Portuguesa, reformado e ampliado em 1889: ligado à colônia de imigrantes portugueses, era destinado aos associados mais pobres e mantido pelas suas elites emergentes, como também o fizeram outras colônias no país (PALMA, 1996). 
Os leprosários são instituições mais antigas, constando que em 1802 a Irmandade da Santa Casa de Misericórdia de São Paulo construiu um pequeno hospital para abrigar os doentes que perambulavam pelas ruas da cidade, e em 1901 o hospital foi transferido para o bairro do Guapira, onde posteriormente foi construído o Leprosário Modelo de Santo Ângelo. Em 1926 já existiam 24 instituições de assistência social e de assistência médica na Capital de São Paulo, dentre eles outros hospitais, e várias instituições de assistência social, confirmando a articulação entre essas áreas no período (PALMA, 1996).

Durante a primeira República também foram criados serviços médicos de urgência para socorro de acidentados na via pública ou no domicílio: constata-se um grande número de atendimentos de vítimas de acidentes do trabalho, agressões físicas e ocorrências nas vias públicas, demonstrando o aumento da violência urbana. Merece referência especial dessa fase $o$ atendimento às vítimas da Revolução de 1924 (PALMA, 1996).

Aspectos importantes a serem ressaltados, foram às tentativas de substituir os inspetores e os fiscais sanitários nos Serviços Sanitários por dois outros profissionais: os médicos sanitaristas e as educadoras sanitárias nos Centros de Saúde, denotando uma nova concepção sobre as doenças e as ações prioritárias para o seu enfrentamento na legislação de 1925, que presidiu a Reforma dos Serviços Sanitários do Estado de São Paulo (MERHY, 1985).

As atividades filantrópicas adquiriram após a Primeira Guerra Mundial uma nova perspectiva: foram intensificadas para enfrentar as agitações políticas do período, com apoio do governo e da Igreja Católica que se propunha a "disputar" com o anarco-sindicalismo e os partidos socialistas e comunistas a liderança das camadas de trabalhadores: ampliaram-se as obras assistenciais, através da criação da Liga das Senhoras Católicas (1923), dos Círculos Operários e da Ação Católica (1928), embasada na Doutrina Social da Igreja e nas encíclicas papais. A Igreja incentivou os leigos a agirem no seu meio social e participou diretamente da formação de intelectuais e profissionais para atuar em função da justiça social, influindo em várias profissões antigas, e, nas novas que iniciaram a sua formação institucionalizada após os anos 30, como ocorreu com o Serviço Social (LIMA, 1991). 


\section{SERVIÇO SOCIAL NA ÁREA DA SAÚDE EM SÃO PAULO: 1940/2000}

A maioria dos TCCs produzidas pelos formandos em Serviço Social desde a fundação da Escola de Serviço Social em 1936, em São Paulo, buscou descrever e analisar a experiência prática de estagiários e assistentes sociais das instituições em que estavam inseridos. Constata-se que no início de sua profissionalização, os assistentes sociais se concentraram em número relevante nos serviços da área de saúde em São Paulo; 23\% dos formados em 1947 pela Escola de Serviço Social de São Paulo estavam atuando no campo do Serviço Social Médico, destacando-se: o Hospital de Clínicas da Faculdade de Medicina da USP e o Hospital da Irmandade da Santa Casa de Misericórdia de São Paulo; acrescente-se o Dispensário de Tuberculose do Departamento de Saúde do Estado, o Departamento Estadual da Criança e outros Hospitais e Clínicas particulares.

Os trabalhos abordaram temáticas relativas à assistência médica e saúde, revelando preocupação com a prática profissional desenvolvida: metodologia, técnicas, instrumentos utilizados na prática cotidiana; certa reflexão sobre a inserção do Serviço Social numa área já existente; e tentativas para dinamizar o acesso e atendimento à saúde, de camadas da população em situação de vulnerabilidade social e de saúde. Além de atuar em hospitais, constituindo o denominado Serviço Social Médico (BEREZOVSKY, 1977), os trabalhos apontam como a profissão foi acompanhando a definição das especialidades médicas que estavam se constituindo no país: Obstetrícia, Pediatria, Ginecologia, Oncologia, Ortopedia; assistência aos portadores de tuberculose, lupus, doenças venéreas, epilepsia, entre outras.

Além do campo hospitalar, registram-se algumas poucas experiências em atividades ambulatoriais e de saúde pública, destacando-se uma no Centro de Saúde Santa Cecília de 1941, em Dispensários de Tuberculose, em Postos de Puericultura,

\footnotetext{
4 “A trajetória do Serviço Social na área da Saúde em São Paulo: 1940/1999”, pesquisa realizada no final dos anos 90, pelos autores AMARAL, D. P.; CARREIRA, M. O. C. S.; FREIRE, V. L.; MARSIGLIA, R.M.G.; MATTINS, G. A. P.; e SETTIN, S.M.C., membros do Grupo de Saúde, vinculados ao Núcleo de Seguridade e Assistência Social do Programa de Estudos Pós-Graduados em Serviço Social da PUCSP. Analisou-se os TCCs produzidos na PUCSP e FMU, de 1940-1999, dissertações/teses defendidas no Programa, que abordaram questões relativas à inserção do serviço na área de saúde, concepções, temáticas abordadas, inserção institucional e tipos de organizações nas quais os assistentes sociais desenvolviam suas práticas, relacionando as especificidades desse processo com os diferentes contextos histórico, social e de saúde presentes na sociedade.
}

Serv. Soc. \& Saúde, Campinas, SP v. 15, n. 1 (21), p. 37-50, jan./jun. 2016 ISSN 1676-6806 
instituições destinadas à atenção para pessoas com deficiência, reabilitação e assistência médica da Previdência Social.

A atuação do assistente social se fazia no sentido de estimular os esforços, as capacidades e a responsabilidade do paciente com o tratamento médico e/ou hospitalar, de intermediar a relação, paciente/ médico/ organização, de dar seguimento ao tratamento médico proporcionando condições para tal. Contribuía ainda para a efetividade da administração hospitalar, desenvolvendo atividades e procedimentos que atualmente são desempenhados por outros profissionais, como Terapia Ocupacional, Relações Públicas, Psicologia, Fisioterapia.

Quanto aos instrumentos técnico-operativos da prática profissional, verificou-se uma priorização das entrevistas como meio de abordagem dos pacientes e suas famílias, visitas domiciliares e a obras sociais, além da produção de relatórios que, deveriam ser anexados aos prontuários médicos.

O processo de crítica teórico-metodológica que perpassou o Serviço Social, tanto na década de 60 como na de 70, não teve grande impacto na sua inserção na área da saúde, mais voltada para a afirmação de um padrão técnico que pudesse dar respostas às exigências das demandas que emergiam da assistência médica, reforçadas no pós 64, apesar de haver alguma sinalização para a área de saúde pública na prevenção, proteção e recuperação da saúde.

A partir dos anos 90, percebe-se o interesse entre os profissionais e futuros assistentes sociais pelas discussões sobre a política de saúde, organização e funcionamento dos serviços de saúde, controle social e educação em saúde, no contexto do Movimento da Reforma Sanitária e implantação do Sistema Único de Saúde (SUS), após a definição da Constituição Federal de 1988. Aparece também preocupação com a questão da "Violência", enquanto problema de saúde, já que seus efeitos refletem-se, tanto na mortalidade, quanto nos serviços de saúde, e, com a AIDS.

É importante considerar que antes da definição do SUS, uma das atribuições importantes do Serviço Social em todos os hospitais públicos, privados, filantrópicos e Hospitais de Clínicas das Universidades que se formaram a partir do final da década de 40, foi a Seleção Socioeconômica dos usuários, para concentrar as atividades de assistência aos que não fossem beneficiários dos Institutos de Aposentadorias e Pensões 
(IAPS), questão discutida por Camilo (1997), na sua análise sobre a universalidade do acesso à saúde no Hospital de Clinicas da Universidade Estadual de Campinas.

Enfatiza-se também nos anos 90, o processo saúde/doença como decorrente das condições de vida e de processos sociais desfavoráveis ou excludentes, como é o caso do alcoolismo, uso de drogas e mortes violentas. O assistente social aparece como membro da equipe multidisciplinar dirigida às ações de prevenção, tratamento e cura do doente e de sua família. Quanto às funções do assistente social, se comparadas às décadas anteriores, pouca coisa se modificou, mas já aparece a necessidade de se potencializar a ação educativa e pedagógica do Serviço Social.

\section{SERVIÇO SOCIAL NA ATENÇÃO PRIMÁRIA EM SAÚDE: MUDANÇAS NAS ADMINISTRAÇÕES PÚBLICAS DE SAÚdE EM SÃO PAULO, A PARTIR DOS ANOS 70}

Durante os anos 70 houve uma reestruturação importante das Secretarias Municipal e Estadual de Saúde de São Paulo. No nível estadual, o processo conhecido como "Reforma Dr. Walter Leser", porque ocorreu em sua gestão, levou à criação de quatro Coordenadorias: de Saúde da Comunidade, de Assistência Hospitalar, de Saúde Mental e de Serviços Técnicos Especializados (IYDA, 1994).

Em todas houve concurso para cargos de assistentes sociais, especialmente na Coordenadoria de Saúde da Comunidade, tanto no nível central da secretaria, como nos centros de saúde da capital e das cidades do interior do Estado de São Paulo: foram criados novos centros de saúde e reestruturados os já existentes, com a implantação dos Programas de Saúde da Criança, da Mulher, Mental, Bucal, de Tuberculose e Hanseníase. Todos passaram a contar com um assistente social para o atendimento dos vários programas e um específico para o programa de Saúde Mental (IYDA, 1994).

No nível municipal, a reforma foi empreendida na gestão do Dr. Cornélio Rosemburgo, quando foi criado o Departamento de Saúde da Comunidade (DSC) para coordenar os Postos de Assistência Médica (PAM), muitos dos quais substituíram os antigos Postos de Puericultura, e passaram a contar com assistentes sociais em seu quadro de pessoal. O mesmo ocorreu com a Superintendência de Administração dos 
Hospitais e Pronto Socorros do município, também com abertura de vagas para assistentes sociais.

Além da atenção direta aos usuários e suas famílias, uma das atribuições definidas para esses profissionais foi articular as atividades dos serviços básicos de saúde, com outras instituições da comunidade ao redor, participar de equipes multiprofissionais e desenvolver atividades de educação em saúde. Em ambas as reformas, houve grande preocupação com as ações de prevenção, vacinação, planejamento dos serviços, definição de rotinas, implantação de prontuários, e em algumas unidades, de prontuários-família, como no caso dos Centros de Saúde ligados às universidades.

Após a implantação do SUS a partir dos anos 90, os Centros de Saúde passaram a denominarem-se Unidades Básicas de Saúde e começaram a ser implantados Conselhos Gestores em cada unidade, na perspectiva de desenvolvimento da participação da população e controle social. Nos níveis municipal e estadual, foram criados os Conselhos Municipais e Estadual de Saúde, cuja composição foi definida como paritária: 50\% de representantes de usuários, e a outra metade de representantes dos trabalhadores de saúde e prestadores de serviços. Muitos desses conselhos contaram com a presença de profissionais de Serviço Social, na qualidade de membros representantes dos trabalhadores ou como assessores, especialmente do segmento de usuários.

Essa situação permaneceu até 1995, quando o Ministério da Saúde passou a implantar o Programa de Saúde da Família, posteriormente denominado Estratégia de Saúde da Família nas Unidades Básicas de Saúde: as Equipes de Saúde da Família, não contaram com a presença de assistentes sociais: apenas médicos, enfermeiras, auxiliares de enfermagem e seis Agentes Comunitários de Saúde (ACS), moradores da área de abrangência da UBS, e que deveriam representar o conhecimento advindo da experiência vivida na comunidade.

Uma década depois, o Ministério da Saúde incentivou a criação dos Núcleos de Apoio às Equipes de Saúde da Família (NASF), e alguns deles passaram a contar na sua composição, com assistentes sociais. Outra diretriz nesse período foi o incentivo a municipalização da Atenção Primária em Saúde: nos vários municípios do Estado de São Paulo, o processo foi se instalando com apoio da SESSP, mas no município da Serv. Soc. \& Saúde, Campinas, SP v.15, n. 1 (21), p. 37-50, jan.jun. 2016 ISSN 1676-6806 
capital, o processo só ocorreu a partir de 2001, porque a capital esteve durante as administrações Maluf e Pitta, sob a regência do Plano de Assistência à Saúde (PAS), tendo por base a formação de cooperativas de profissionais e não a prestação direta do serviço pela poder público.

Atualmente, as UBS no município de São Paulo estão organizadas sob três modelos: dos programas de saúde implantados nos anos 70, que denominamos de Ação Programática e que contam com assistentes sociais em seu quadro; da Estratégia de Saúde da Família, sem assistentes sociais, a não ser no NASF e as UBS "Mistas", em que coexistem os dois modelos de Ações Programáticas e Saúde da Família, e em se encontram assistentes sociais, originários do modelo de Ação Programática.

\section{PRODUÇÃO E PUBLICIZAÇÃO DO TRABALHO E REFLEXÕES DOS ASSISTENTES SOCIAIS DA ÁREA DA SAÚDE}

Na segunda metade dos anos 90, os assistentes sociais dos serviços ligados às Universidades de São Paulo (USP), Universidade Estadual de Campinas (UNICAMP) e Universidade Estadual Paulista (UNESP), passaram a organizar Simpósios do Serviço Social em Saúde, apresentando seus trabalhos e experiências nos vários serviços ligados a essas universidades, tendo em 2014 realizado o sétimo evento. Esses eventos permitiram discutir a inserção da profisssão nos hospitais universitários/ensino, analisar as concepções dominantes na profissão e na área de saúde, ao lado da contribuição de seus intelectuais, visando repensar o trabalho futuro.

Destacamos o II Simpósio, realizado em 1998 em São Paulo, com a temática "Novas Demandas Sociais: posicionamento das instituições e o Serviço Social”, que se propôs a criar um "espaço de reflexão e debates quanto ao posicionamento da sociedade, das instituições e dos profissionais de saúde, frente às demandas da sociedade" naquele contexto do país. E o III Simpósio, realizado em Campinas, em 2000, tendo como eixo central o "Serviço Social, Saúde e Sociedade: do Político ao Pontual". O evento apontou a preocupação dos profissionais com "as políticas sociais, mudanças no mundo do trabalho, missão social da universidade, bioética, equidade e respostas dadas pelo Serviço Social nesta sociedade em transformação, através de suas práticas".

Serv. Soc. \& Saúde, Campinas, SP v. 15, n. 1 (21), p. 37-50, jan./jun. 2016 ISSN 1676-6806 
Em 2002 foi lançado o primeiro número da Revista Serviço Social \& Saúde da Universidade Estadual de Campinas, em comemoração aos 35 anos da UNICAMP e 35 anos do Serviço Social em suas dependências: HC, CAISM, CEPRE, CECOM, SAE, DAB, FOP, HEMOCENTRO e CECI. Segundo seu Conselho Editorial, a Revista "surgiu da necessidade de situar a trajetória da profissão na universidade, implantada junto à Faculdade de Ciências Médicas [...] pontuando seus avanços técnicos e científicos, o compromisso ético-político com os direitos de cidadania, expandindo-se a todas as unidades de saúde e de atenção a alunos e funcionários" (Serviço Social \& Saúde, 2002, p. v).

Em 2004, a assinatura de um Convênio de Cooperação Técnica entre o Ministério da Saúde e a Associação Brasileira de Pesquisa em Serviço Social (ABEPSS), através do projeto Serviço Social: interfaces com a saúde: a) viabilizou a realização de atividades de formação e capacitação profissional na área do Serviço Social, nas seis regionais da ABEPSS; b) incentivou a mudanças nos cursos de graduação das profissões que são da área da saúde ou que tem interfaces com a pesquisa, tendo em vista a formulação e implantação do SUS, como é o caso do Serviço Social; c) mobilizou associações e grupos profissionais para participarem desses processos; d) publicou uma Coletânea (MOTA et al., 2006, p. 7-8).

A Coletânea Serviço Social e Saúde: Formação e Trabalho Profissional, MOTA et al, com apoio da OPAS, OMS, M.SAÚDE. São Paulo: EDITORA CORTEZ, 2006, com várias reedições, foi organizada em três partes.

1a. Parte: Política Social e Política de Saúde, com artigos de Elaine Rossetti Behring, Ana Elisabete Mota, Ivanete Boschetti e Evilásio Salvador, Raquel Raichelis, Maria Inês Souza Bravo e Maria Valéria Costa Correia.

2. Parte: Serviço Social e Saúde, com artigos de José Paulo Netto, Marilda Villela Iamamoto, Maria Inês Souza Bravo e Maurílio Castro de Matos, Vera Maria Ribeiro Nogueira e Regina Célia Tamaso Mioto, Ana Maria de Vasconcelos, Maria Dalva Horácio da Costa, Ana M. Arreguy Mourão, Ana M.C. Amoroso Lima, Ayta I. Stephan- Souza e Leda M. Leal de Oliveira.

3. Parte: Pesquisa e Sistematização do Trabalho Profissional: com artigos de Regina Maria Giffoni Marsiglia e Ney Luiz Teixeira de Almeida. 


\section{CONSIDERAÇÕES FINAIS}

O Serviço Social englobou uma variada gama de temáticas na área de saúde em São Paulo, na medida em que acompanhou a dinâmica de desenvolvimento da área, o que permite afirmar que durante os anos estudados, os assistentes sociais tiveram seu mercado de trabalho ampliado na área. Sua inserção inicial ocorreu nos hospitais filantrópicos, especialmente na Santa Casa de Misericórdia de São Paulo, e a partir da expansão das faculdades de Medicina e Enfermagem de caráter público, tanto na capital quanto em outros municípios do interior do Estado de São Paulo, os profissionais passaram a integrar o corpo de trabalhadores dos hospitais universitários e de ensino, denominando-se Serviço Social Médico. Ao mesmo tempo a profissão foi sendo incorporada nas primeiras unidades de atendimento ambulatorial destinadas aos problemas coletivos de saúde, ligadas à Secretaria de Estado da Saúde, desde o início dos anos 1940.

A expansão da rede básica de saúde no Estado e no Município de São Paulo, a partir das reformas dessas administrações nos anos de 1970, abriu novas oportunidades de trabalho do Serviço Social na Saúde. Trajetória esta, diferente da profissão no Rio de Janeiro, que como demonstrou Bravo (1996), apresentou uma inserção bastante significativa nos serviços ligados à Previdência Social. A trajetória da profissão na área de saúde em São Paulo, do ponto de vista da expansão e diversificação de seu mercado de trabalho, acompanhou a dinâmica da expansão e desenvolvimento das especialidades médicas na área hospitalar, e da atenção primária em saúde.

Embora como categoria profissional, não tenha tido presença ativa no Movimento da Reforma Sanitária ao final dos anos 70 e durante os anos 80, após a definição do Sistema Único de Saúde na Constituição Federal de 1988, parte da categoria vem contribuindo para as discussões e propostas de implantação desse sistema universal. Tem se envolvido nas lutas pelos direitos de acesso aos serviços, equidade e controle social, em conformidade com os contextos sociais e políticos do Estado e Municípios de São Paulo, e as determinações sociais mais amplos, da política de saúde em nosso país. 


\section{REFERÊNCIAS}

BEREZOVSKY, M. Serviço Social Médico na Administração Hospitalar, São Paulo, Editora Cortez \& Moraes, 1977.

BRAGA, J.C.; PAUlA, S.G. Saúde e Previdência: Estudos de Política Social, São Paulo, CEBES-Hucitec, 1981.

BRAVO, M.I.S. Serviço Social e Reforma Sanitária: Lutas Sociais e Práticas Profissionais. Rio de Janeiro: Ed. UFRJ; São Paulo: Cortez Editores, 1996.

CAMILO, M.V.R. F. A Universalidade de Acesso enquanto Expressão do Direito à Saúde: a trajetória histórica do Hospital das Clínicas da Unicamp de 1966/96. 1997. Dissertação (Mestrado em Serviço Social) - Pontifícia Universidade Católica de São Paulo (Biblioteca).

IAMAMOTO, M. V.; CARVALHO, R. Relações Sociais e Serviço Social no Brasil. São Paulo, Celats/Cortez, 1982.

IAMAMOTO, M.V. O Serviço Social na Contemporaneidade: trabalho e formação profissional. $3^{\text {a }}$ ed. São Paulo, Cortez, 2000.

IYDA, M. Cem Anos de Saúde Pública: a cidadania negada. São Paulo, Prismas UNESP, 1994.

MERHY, E. E. O Capitalismo e a Saúde Pública: a emergência das práticas sanitárias no Estado de São Paulo. São Paulo/Campinas. Ed. Papirus, 1985.

MOREIRA LIMA, V.L.F. Um início do Serviço Social no Brasil: um feminismo cristão. Dissertação de Mestrado. Programa de Pós Graduação em Serviço Social Pontifícia Universidade Católica de São Paulo. 1991. (Biblioteca).

MOTA, A. E.; BRAVO, M.I.S.; UCHÔA, R., NOGUEIRA, V.M.R., MARSIGLIA, R.M.G., GOMES, L., TEIXEIRA, M. (Orgs.) Serviço Social e Saúde: formação e trabalho profissional. OPAS, OMS, MINISTÉRIO da SAÚDE. São Paulo: CORTEZ EDITORA, 2006.

NETTO, J. P. Ditadura e Serviço Social: uma análise do Serviço Social no Brasil pós 64. São Paulo, Cortez, 1999.

PALMA, J. Os trabalhadores em saúde: agentes, práticas e instituições de saúde em São Paulo. Tese de Doutorado. Faculdade de Medicina da USP. Departamento de Medicina Preventiva. 1996. (Biblioteca) 
SERVIÇO SOCIAL \& SAÚDE, Universidade Estadual de Campinas. Hospital de Clínicas, Serviço Social. Campinas, SP, v. 1, n. 1, 2002. Campinas, maio 2002. ISSN 1676-6806.

SIMPÓSIOS DE SERVIÇO SOCIAL EM SAÚDE. USP, UNICAMP e UNESP (Org.)

Novas demandas sociais: posicionamento das Instituições e o Serviço Social (1998).

SIMPÓSIOS DE SERVIÇO SOCIAL EM SAÚDE. USP, UNICAMP e UNESP (Org.) Serviço Social, Saúde e Sociedade: do Político ao Pontual (2000). 